\title{
THE DISPERSION OF ELECTRIC DOUBLE REFRACTION.
}

By T. H. Havelock.

A RECENT paper by C. F. Hagenow, ${ }^{1}$ on electric double reA fraction in carbon bisulphide suggests a comparison of the experimental results with a formula which the present writer has given connecting the dispersion of artificial double refraction with the ordinary dispersion of the isotropic medium. ${ }^{2}$ In this connection the theory may be indicated briefly in the following manner.

In any medium the total electric displacement consists of two parts : (I) the æthereal displacement, measured by $(4 \pi)^{-1}$ times the electric force, (2) the material polarization of the particles constituting the medium. The latter part depends upon the effective electric force acting upon the particles, and this in turn depends for each particle upon the character and arrangement of the surrounding particles. We imagine an effective cavity to be associated with each particle, such that the force on the particle is the force within such a cavity in a medium uniformly polarized to the value of the material polarization vector at the point in question. For an isotropic medium the effective cavity is taken to be a sphere; as is well known, this leads to the formula

$$
\frac{n_{0}^{2}-\mathrm{I}}{n_{0}^{2}+2}=\frac{4 \pi}{3} \Sigma_{h} \frac{\alpha_{h}}{k_{h}^{2}-k^{2}},
$$

where $n_{0}$ is the refractive index of the medium, $2 \pi / k$ is the period of the vibrations, and $\alpha_{h}, k_{h}$ are constants assigned to each type of particle, the summation being extended over the various types. Suppose that the medium is subjected to mechanical stress, or to the action of an electric or magnetic field, so that it becomes doubly refracting; assume further that the consequent slight modification of the optical properties can be represented by a change of the effec-

${ }^{1}$ C. F. Hagenow, Physical Review, vol. 27, p. 196, 1908.

2 T. H. Havelock, Proc. Roy. Soc., A., vol. 8o, p. 28, 1907. 
tive cavity from a sphere to an ellipsoid. Let the axes of the ellipsoid be in the ratios of $\mathrm{I}+\varepsilon_{1}, \mathrm{I}+\varepsilon_{2}$ and $\mathrm{I}+\varepsilon_{3}$; also let $n_{1}, n_{2}, n_{3}$ be the principal refractive indices of the medium.

The components of effective force within the cavity can be evaluated and we obtain, instead of ( 1 ), three equations of the type

where

$$
\frac{n_{1}{ }^{2}-\mathrm{I}}{n_{1}^{2}+2+3 s_{1}\left(n_{1}^{2}-\mathrm{I}\right)}=\frac{4 \pi}{3} \dot{\nu}_{k}^{\prime} \frac{\alpha_{h}}{k_{k}^{2}-k^{2}}
$$

$$
S_{1}=-\frac{4}{\mathrm{I} 5} \varepsilon_{1}+\frac{2}{\mathrm{I} 5}\left(\varepsilon_{2}+\varepsilon_{3}\right) \text {. }
$$

Hence from (I) and (2) we can find expressions for the principal indices in terms of $n_{0}$, the refractive index of the isotropic medium composed of the same particles in equal density.

If the strained medium behaves like a uniaxial crystal, we have

$$
\begin{aligned}
& n_{1}=n_{0}-\frac{2}{\mathrm{I} 5} \frac{\left(n_{0}{ }^{2}-\mathrm{I}\right)^{2}}{n^{0}} \varepsilon \\
& n_{2}=n_{0}+\frac{\mathrm{I}}{\mathrm{I} 5} \frac{\left(n_{0}{ }^{2}-\mathrm{I}\right)^{2}}{n_{0}} \varepsilon .
\end{aligned}
$$

Hence the dispersion of the double refraction, measured by the difference of the ordinary and extraordinary indices, is given by

$$
\delta \infty \frac{\left(n_{0}^{2}-1\right)^{2}}{n_{0}}
$$

This formula connects the dispersion of the slight artificial double refraction with the ordinary dispersion of the isotropic medium. The relation suggested by Kerr as the result of experiments was, that the double infraction induced in $\mathrm{CS}_{2}$ by an electric field was inversely proportional to the square root of the wave-length, but this has been disproved by the experiments of Blackwell, ${ }^{1}$ and again by those of Hagenow. In the paper already quoted, the writer has shown that the formula (3) agrees well with Blackwell's results; this is also the case for the more recent data, as is shown by the following table. The first two columns give the experimental data which are taken from Hagenow's paper; these are the average results of sets of observations and it is claimed that the experimental error is small,

${ }^{1}$ H. L. Blackwell, Proc. Amer. Acad., vol. 41, p. 650, 1906. 
except that the value of $\delta$ for $\lambda$ equal to 600 appears to be abnormally low and the value of $\lambda$ equal to 700 is also not so reliable. The values for $n_{0}$ given in the third column were obtained by the help of the table given by Martens, ${ }^{1}$ using also a temperature correction of $-9 \times 10^{-4}$ for each degree rise in temperature; we obtain thus values correct to three decimal places. The fourth column shows the values of $\delta n_{0} /\left(n_{0}{ }^{2}-\mathrm{I}\right)^{2}$, which ought to be constant by relation (3); omitting the values at 600 and 700 for the reasons stated above, we have a mean value of 60.57 for the remaining six values, showing a maximum divergence from the mean of about .6 per eent.

\begin{tabular}{c|c|c|c}
\multicolumn{3}{|c|}{$C S_{2}$ at $25^{\circ}}$. \\
\hline$x$ & $\delta$ obs. & $n_{0}$ & $\delta n_{0} /\left(n_{0}{ }^{2}-1\right)^{2}$ \\
\hline 430 & 116.2 & 1.672 & 60.27 \\
450 & 112.5 & 1.662 & 60.22 \\
500 & 106.8 & 1.643 & 60.75 \\
550 & 102.1 & 1.630 & 60.60 \\
590 & 100 & 1.623 & 60.78 \\
600 & 97.5 & 1.621 & 59.68 \\
650 & 97.1 & 1.614 & 60.83 \\
700 & 96.7 & 1.610 & 61.42 \\
\hline
\end{tabular}

A preliminary account of investigations on the magnetic and electric double refraction of nitrobenzene has recently been published by Cotton and Mouton. ${ }^{2}$ This liquid is interesting on account of the double refraction being extremely large compared with that of $\mathrm{CS}_{2}$; a series of observations is given showing the magnetic double refraction at various wave-lengths and it is stated that electric double refraction of the same order was obtained. Owing to the present lack of more exact data both for the double refraction and for the ordinary dispersion of nitrobenzene under similar conditions it is not possible to compare the formula given above with these results.

As a result of their series of experiments on magnetic double refraction, dealing originally with the Mayorana effect in colloidal solutions, Cotton and Mouton believe that the phenomena are due to orientation of particles, and they suggest a similar explana-

${ }^{1}$ Martens, Annalen der Physik, vol. 6, p. 632, 1901.

${ }^{2}$ Cotton and Mouton, Comptes Rendus, t. 147, p. 193, 1908. 
No. 2.] DISPERSION OF ELECTRIC DOUBLE REFRACTION. I 39

tion for the electric double refraction in nitrobenzene, namely, orientation of the molecules. In the theory sketched above, an essential point is in regarding the effective cavity to be changed from a sphere to an ellipsoid by the impressed field. Now this may be due to an orientation of similar æolotropic particles, if we regard the isotropy of the unstrained medium as an average effect of orientation of the particles in all possible directions ; or it may be due to a rearrangement in grouping of an assemblage of similar particles. In deducing the relation (3) given above, the latter hypothesis was used, and we have seen that the formula is supported by the experimental data available at present.

Whether a similar formula could be obtained on the hypothesis of orientation or not, it seems desirable to have as far as possible parallel series of observations on the dispersion of the artificial double refraction and the ordinary dispersion of the isotropic medium taken under similar conditions, for instance as regards temperature and purity of materials.

Armstrong College, Newcastle-on-Tyne, November 6, 1908. 